\title{
A Method for Reduced Finite Precision Effects in Parallel Filtering Echo Cancellation
}

\author{
Fredric Lindstrom, Member, IEEE, Christian Schüldt, Student Member, IEEE, Mikael Långström, and \\ Ingvar Claesson, Member, IEEE
}

\begin{abstract}
The two-path algorithm is an adaptive filter algorithm based on a parallel filter structure, which has been found to be useful for line echo cancellation as well as for acoustic echo cancellation. It is well known that in finite precision arithmetic, the adaptation process of adaptive algorithms can be reduced or even halted due to finite precision effects. This paper proposes a variant of the two-path scheme where the effects of quantization are reduced, without any significant increase in complexity. The improvement is shown by simulations using bandlimited flat spectrum noise as well as real speech signals.
\end{abstract}

Index Terms-Adaptive filtering, echo cancellation, fix-point arithmetic, parallel filters, quantization.

\section{INTRODUCTION}

$\mathbf{T}$ HE two-path algorithm [1], originally proposed for robust line echo cancellation, has previously been extended to several alternative echo cancellation applications [2]-[9]. This has been achieved through alternative update control logic [2], [3]. The two-path structure for acoustic echo cancellation was introduced in [4], with an extension to stereo acoustic cancellation in [5]. The two-path structure is presented in a doubletalk detector setup in [6]. In such a structure, the two-path scheme is used to improve the performance of doubletalk detectors (e.g., [7]) or as a rescue scheme in adaptive step-size configurations [8]. An overview of some different two-path configurations is provided in [9].

The basic two-path algorithm structure consists of two parallel adaptive filters, commonly denoted as the background filter and the foreground filter. The first (background) filter is continuously adapted, while the second (foreground) filter is mostly kept in a fixed state. The performance of the background filter is continuously compared to that of the foreground filter and when the background filter is considered to yield a better estimation of the echo, the foreground filter is updated with the coefficients of the background filter. This procedure allows a structure which is robust to doubletalk disturbance and also avoids unnecessary halting of the adaptation process. In this paper, we consider a setup where the popular normalized LMS (NLMS) is used for the adaptation process. However, the proposed scheme is not limited to the NLMS, but can be used in conjunction with other

Manuscript received October 11, 2006; revised March 15, 2007. This work was supported by the Swedish Knowledge Foundation (KKS).

F. Lindstrom and C. Schüldt are with Konftel, Umea SE-901 06, Sweden (e-mail: fli@konftel.com).

M. Långström is with Fält Communications, Umea SE-906 20, Sweden.

I. Claesson is with Blekinge Institute of Technology, Ronneby SE-372 25, Sweden.

Digital Object Identifier 10.1109/TCSI.2007.904664 adaptation methods (e.g., affine projection-type algorithms or recursive least squares).

When implemented in finite precision arithmetic, LMS-based algorithms (such as the NLMS) might suffer from performance degradation due to quantization effects [10]. The quantization of the LMS might lead to a halt of adaptation, a so-called stalling phenomenon, as first demonstrated in [11]. Further analysis has shown the effects on steady-state mean square error [12] as well as demonstrating that the stalling is actually an extreme slowdown of the algorithm [13], [14]. Two general approaches have been proposed to avoid, or reduce, the effects of stalling [10] — either limit the lowest possible value for the step-size control $\mu$ or increase the number of bits. The general design approach is to, for a given bit precision, determine whether optimal settings of the step-size control parameter $\mu$ can meet the design requirements (e.g., the required steady-state mean square error). If this cannot be achieved, the bit precision needs to be increased [15]. Increasing the number of bits might lead to an increased silicon surface, cost, and/or battery consumption. In [16], a scheme was proposed that uses two parallel filters operating in different bit ranges. This paper elaborates the idea in [16] by modifying it into a two-path scheme. Further, this paper proposes a control algorithm that adaptively determines the range of the background filter depending on the convergence of the foreground filter. The proposed scheme reduces the quantization effects, resulting in increased echo cancellation performance, without introducing any significant increase in computational complexity.

\section{Two-Path AlgORIthm}

In an echo cancellation scheme based on adaptive filtering, it is essential that the adaptive filter not be updated during doubletalk (i.e., when both talkers are active simultaneously [9]). Updating the filter in such a situation might lead to filter divergence and, thus, poor cancellation or even howling. However, introducing this type of mechanism induces the risk of unnecessary halting of the adaptation which, in turn, leads to slower convergence. In the two-path algorithm, as depicted in Fig. 1, the risk of unnecessary halting is avoided thanks to the continuously updating background filter.

The variant of the two-path algorithm addressed in this paper is used in an acoustic echo cancellation (AEC) context, although the procedure is applicable for line echo cancellation as well. In the acoustic echo cancellation case, the echo path consists of the loudspeaker-enclosure-microphone (LEM) system, whereas in the case of line echo cancellation, the echo path corresponds to the 2/4-wire hybrid. 


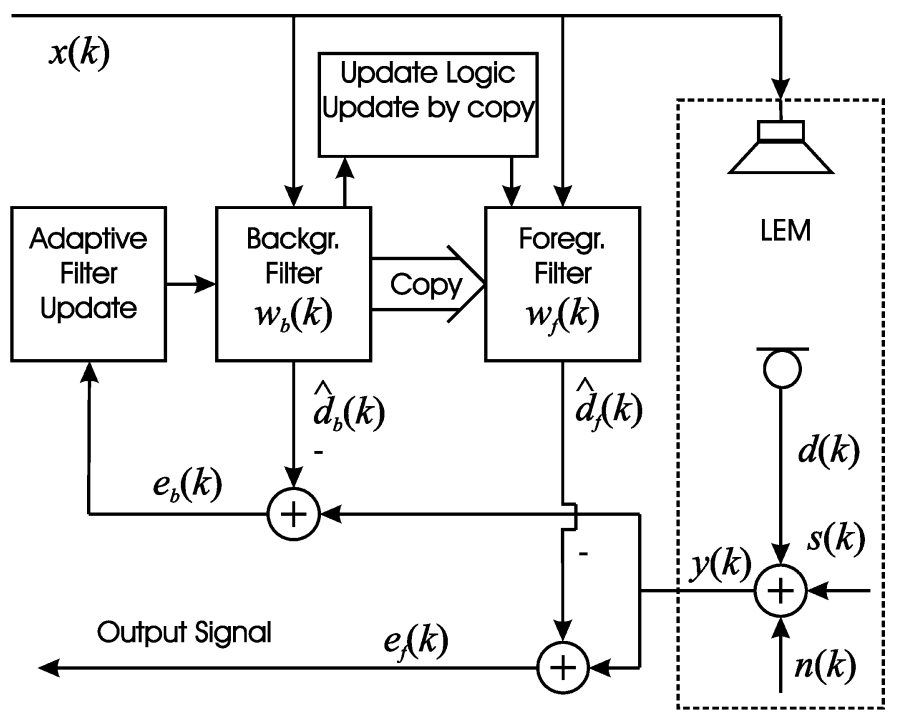

Fig. 1. Two-path scheme.

The loudspeaker signal $x(k)$ (see Fig. 1) generates an output in the form of an acoustic echo signal, (the "desired" signal in system identification terminology) $d(k)$, where $k$ is the sample index. The microphone signal $y(k)$ consists of the acoustic echo, the background noise $n(k)$, and possible near-end speech $s(k)$ (i.e., $y(k)=d(k)+n(k)+s(k))$. A finite impulse response (FIR) filter of length $N$ is used as a foreground filter $\mathbf{w}_{f}(k)$ (i.e., $\left.\mathbf{w}_{f}(k)=\left[w_{f, 0}(k), \ldots, w_{f, N-1}(k)\right]^{T}\right)$. The foreground filter produces an estimate $\hat{d}_{f}(k)$ of the acoustic echo, which is subtracted from the microphone signal in order to obtain an echo-cancelled "error" signal

$$
e_{f}(k)=y(k)-\hat{d}_{f}(k)=y(k)-\mathbf{w}_{f}(k)^{T} \mathbf{x}(k)
$$

where $\mathbf{x}(k)=[x(k), \ldots, x(k-N+1)]^{T}$. Analogously, for the background filter, we obtain

$$
e_{b}(k)=y(k)-\hat{d}_{b}(k)=y(k)-\mathbf{w}_{b}(k)^{T} \mathbf{x}(k)
$$

where $\mathbf{w}_{b}(k)=\left[w_{b, 0}(k), \ldots, w_{b, N-1}(k)\right]^{T}$.

The NLMS algorithm is used to update the background filter according to

$$
\mathbf{w}_{b}(k+1)=\mathbf{w}_{b}(k)+\frac{\mu e_{b}(k) \mathbf{x}(k)}{\|\mathbf{x}(k)\|^{2}+\epsilon}
$$

where $\mu$ is the step-size control parameter, $\|\mathbf{x}(k)\|^{2}=$ $\mathbf{x}(k)^{T} \mathbf{x}(k)$ is the squared Euclidian norm, and $\epsilon$ is the regularization parameter [10].

The coefficients of the foreground filter $\mathbf{w}_{f}(k)$ are updated with the coefficients of the background filter $\mathbf{w}_{b}(k)$ when the background filter is estimated to perform better in terms of echo cancellation. This update is controlled by update logic (UL) according to

$$
\mathbf{w}_{f}(k+1)= \begin{cases}\mathbf{w}_{b}(k), & \text { if UL indicates update } \\ \mathbf{w}_{f}(k), & \text { otherwise. }\end{cases}
$$

Typically, this update check is not performed for every sample, but at regular intervals in order to reduce complexity.
An example of update logic for line echo cancellation is presented in [1] and [6], which basically allows updating of the foreground filter when all conditions in (5) are true

$$
\frac{P_{y}(k)}{P_{x}(k)}<T_{y, x}, \quad \frac{P_{e_{b}}(k)}{P_{y}(k)}<T_{e_{b}, y}, \quad \frac{P_{e_{b}}(k)}{P_{e_{f}}(k)}<T_{e_{b}, e_{f}}
$$

where $T_{y, x}, T_{e_{b}, y}$, and $T_{e_{b}, e_{f}}$ are thresholds and $P_{[\cdot]}(k)$ denotes a short-time power estimate. Other suggestions of update logics can be found in, for example, [2], [3], [7], [8].

In this paper, however, no specific update logic is studied, as it is assumed that the update logic is operating correctly (i.e., an update is indicated at all times except during doubletalk).

The two-path scheme as depicted in Fig. 1 and described through (2)-(4) is denoted as the conventional two-path algorithm.

\section{Finite PRECISION NLMS Algorithm}

In this paper, a binary number representation using the fix-point two's-complement format [17], with number range $[-1,1)$, is assumed. The quantized $q$-bit precision representation $a_{q}$ of an arbitrary value $a$ is given by $a_{q}=Q_{q}[a]$, where the subindex $q$ denotes the bit precision and the quantization function $Q_{q}[\cdot]$ is defined through

$$
Q_{q}[a]=\left(-b_{0}+\sum_{i=1}^{q-1} b_{i} 2^{-i}\right)
$$

$b_{i} \in\{0,1\}, i=0, \ldots, q-1$, and the values of the elements $b_{i}$ are chosen so that they minimize the expression $\left|a-Q_{q}[a]\right|$.

A product or a division of two arbitrary $q$-bit precision numbers suffers from quantization effects, while a sum has no quantization providing that no overflow occurs. The $q$-bit finite precision NLMS algorithm is thus given by

$$
\begin{aligned}
e_{q}(k) & =y_{q}(k)-\sum_{i=0}^{N-1} Q_{q}\left[w_{q, i}(k) x_{q}(k-i)\right] \\
\beta_{q}(k) & =Q_{q}\left[\mu Q_{q}\left[\frac{e_{q}(k)}{\left\|\mathbf{x}_{q}(k)\right\|_{q}^{2}+\epsilon_{q}}\right]\right] \\
\mathbf{w}_{q}(k+1) & =\mathbf{w}_{q}(k)+Q_{q}\left[\beta_{q}(k) \mathbf{x}_{q}(k)\right] .
\end{aligned}
$$

Many finite precision systems allow vector inner product operations to be carried out in a higher precision, making the quantization of element multiplications insignificant (i.e., the expres$\operatorname{sion} \sum_{i=0}^{N-1} Q_{q}\left[w_{q, i}(k) x_{q}(k-i)\right]$ can be replaced with approximately $\mathbf{w}_{q}(k)^{T} \mathbf{x}_{q}(k)$ if the final scalar result is stored in a higher precision). Further, the calculations in (7) and (8) can also be performed in higher precision which further reduces the quantization effects in these equations. Thus, a less quantized NLMS algorithm can be evaluated as

$$
\begin{aligned}
e(k) & =y_{q}(k)-\mathbf{w}_{q}(k)^{T} \mathbf{x}_{q}(k) \\
\beta_{q}(k) & =Q_{q}\left[\frac{\mu e(k)}{\left\|\mathbf{x}_{q}(k)\right\|^{2}+\epsilon}\right] \\
\mathbf{w}_{q}(k+1) & =\mathbf{w}_{q}(k)+Q_{q}\left[\beta_{q} \mathbf{x}_{q}(k)\right] .
\end{aligned}
$$

Note that in (11), the step-size parameter $\beta_{q}(k)$ is quantized into $q$-bit precision. This is done in order to perform the $q$ by 
$q$-bit multiplications in (12). The increased complexity from the higher precision calculation is generally insignificant since the filtering and updating are more demanding, with the filtering length $N>1000$ in a typical AEC application.

In this paper, the quantized NLMS implementation as given in (10)-(12) is used. (Evaluating the proposed scheme using a more quantized NLMS implementation as in (7) - (9) will likely yield even more positive effects of the proposed improvement).

The finite precision implementation of the NLMS algorithm might lead to stalling, which is when the filter convergence is reduced or even stopped due to some or all of the values of the update vector $Q_{q}\left[\beta_{q}(k) \mathbf{x}_{q}(k)\right]$ being less than the least-significant bit (LSB) [10]. Stalling of the background filter implies that the low-order bits in the coefficients of the foreground filter $\mathbf{w}_{f}(k)$ will not be tuned to their optimal value. If these bits are significant for the estimation of the acoustic echo, the stalling will lead to poor cancellation of the echo in the output signal $e_{f}(k)$.

\section{PROPOSED SCHEME}

When the foreground filter has converged, there is a redundance in the parallel evaluation of the two filters (i.e., after convergence, the high-order bits in the background and the foreground filters are the same). The idea behind the proposed algorithm is to use this redundancy in order to improve performance of the two-path algorithm without increasing the complexity.

This paper proposes a scheme where the output of the foreground filter is used as an input to the background filter (see Fig. 2), and the update by copying the background filter to the foreground filter and is replaced by updating the foreground filter by the sum of the background and foreground filter. This scheme allows an adaptive gain to be implemented in the signal path of the background filter which, in turn, can be used to alter the bit range of the background filter. This paper also proposes a control algorithm for this adaptive gain. The proposed scheme does not imply any significant increase in complexity and the additional control mechanisms required are relatively easy to tune.

The proposed scheme is depicted in Fig. 2. All variables and parameters associated with the proposed scheme are denoted with an additional subindex index $p$ (e.g., the two-path foreground filter in the proposed scheme is denoted as $\mathbf{w}_{f_{p}}(k)$ ), the microphone signal is denoted by $y_{p}(k)$, etc. In most of the equations describing the proposed algorithm, the quantization function and the bit precision subindex has been omitted for clarity of presentation.

As can be seen in Fig. 2, the output of the foreground filter

$$
e_{f_{p}}(k)=y_{p}(k)-\hat{d}_{f_{p}}(k)=y_{p}(k)-\mathbf{w}_{f_{p}}(k)^{T} \mathbf{x}_{p}(k)
$$

is multiplied with a factor $2^{g(k)}$, where $g(k)$ is a nonnegative integer (i.e., the gain operation is implemented as a bitwise shift). The use of this shift operation facilitates the implementation in a fix-point environment. The shifted foreground filter output is denoted as $y_{b_{p}}(k)$, with

$$
y_{b_{p}}(k)=2^{g(k)} e_{f_{p}}(k) .
$$

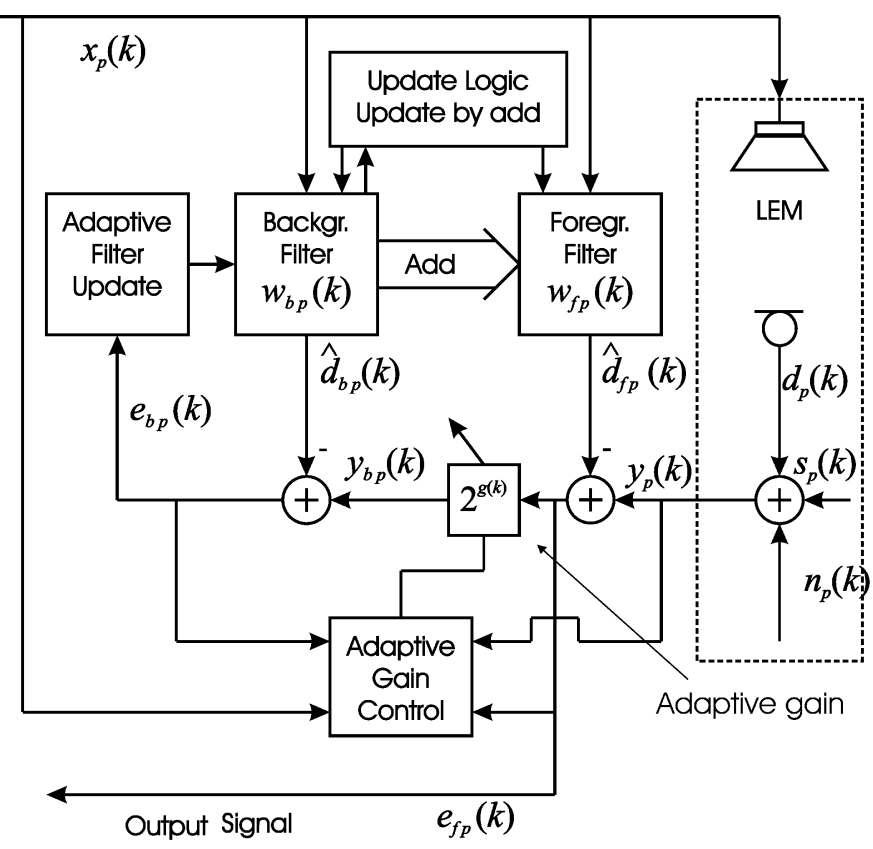

Fig. 2. Proposed two-path scheme.

The error signal of the background filter $e_{b_{p}}(k)$ is formed by subtracting the background filter output from the gained foreground filter output, according to

$$
e_{b_{p}}(k)=y_{b_{p}}(k)-\hat{d}_{b_{p}}(k)=y_{b_{p}}(k)-\mathbf{w}_{b_{p}}(k)^{T} \mathbf{x}_{p}(k) \text {. }
$$

The background filter $\mathbf{w}_{b_{p}}(k)$ is updated using the NLMS, but with $e_{b_{p}}(k)$ as given in (15).

Assume that $g(k)=G$, where $G$ is a fixed positive integer constant. This implies a $G$-bit upshift of the input signal $y_{b_{p}}(k)$. An upshift of $y_{b_{p}}(k)$ will lead to a corresponding upshift of the filter coefficients in $\mathbf{w}_{b_{p}}(k)$. The effects of shifting up a value prior to quantization is equivalent to decreasing the number of bits lost due to quantization, assuming no overflow occurs, i.e.,

$$
Q_{q}\left[2^{G} \mathbf{w}_{b_{p}}(k)\right]=2^{G} Q_{q+G}\left[\mathbf{w}_{b_{p}}(k)\right] .
$$

Thus, selecting $g(k)>0$ is equivalent to increased NLMS quantizing precision with $g(k)$-bits, which means that the impact of stalling is reduced.

When the foreground filter is to be updated, the different bit ranges of the two filters must be accounted for. This is achieved by shifting down the coefficients of the background filter $g(k)$-bits. Thereafter, the updated foreground filter is constructed by adding each shifted background filter coefficient to its corresponding foreground filter counterpart. Finally, the background filter coefficients are reset to zero. Thus, the proposed scheme does not increase the number of bits in the foreground filter, but by letting the background filter operate in an adaptive bit range, the proposed scheme allows the least-significant bits in the foreground filter to converge. This leads to better echo cancellation compared to the conventional two-path solution 
The update check is performed every $M$ sample (i.e., at regular intervals as described in Section II). Thus, if the UL indicates update, the foreground filter update is given by

$$
\mathbf{w}_{f_{p}}(k)=\mathbf{w}_{f_{p}}(k-1)+2^{-g(k)} \mathbf{w}_{b_{p}}(k)
$$

and the background filter is given by

$$
\mathbf{w}_{b_{p}}(k)=\mathbf{0}
$$

where $\mathbf{0}$ is a zero vector of length $N$. An alternative solution is to update the background filter as

$$
\mathbf{w}_{b_{p}}(k)=\mathbf{w}_{b_{p}}(k)-Q_{q-g(k)}\left[\mathbf{w}_{b_{p}}(k)\right]
$$

i.e., keeping the low bits that are not added into the foreground filter.

If the UL does not indicate an update, the foreground filter is unchanged

$$
\mathbf{w}_{f_{p}}(k)=\mathbf{w}_{f_{p}}(k-1)
$$

and the background filter is updated according to the regular NLMS

$$
\mathbf{w}_{b_{p}}(k)=\mathbf{w}_{b_{p}}(k-1)+\frac{\mu e_{b_{p}}(k) \mathbf{x}_{p}(k)}{\left\|\mathbf{x}_{p}(k)\right\|^{2}+\epsilon}
$$

where $\left\|\mathbf{x}_{p}(k)\right\|^{2}$ is recursively calculated as

$$
\left\|\mathbf{x}_{p}(k)\right\|^{2}=\left\|\mathbf{x}_{p}(k-1)\right\|^{2}+x_{p}^{2}(k)-x_{p}^{2}(k-N)
$$

in order to reduce complexity.

The shift integer $g(k)$ should initially be set to zero and increase as the background filter converges. Basically, $g(k)$ could be increased with 1 for every high-order bit in the foreground filter which has converged, for example, if the most significant bit of all coefficients in the foreground filter has reached a stable nonchanging value, $g(k)$ could be increased from 0 to 1 without risking the background filter overflow. Thus, the gain $g(k)$ should be set with respect to the current echo return loss enhancement (ERLE) achieved with the foreground filter

$$
\operatorname{ERLE}(k)=\frac{E\left\{d_{p}^{2}(k)\right\}}{E\left\{\left(d_{p}(k)-\hat{d}_{f_{p}}(k)\right)^{2}\right\}},
$$

where $E\{\cdot\}$ denotes the expected value.

Obviously, an estimation of ERLE should only be performed when far-end speech is present. This can be guaranteed by a simple activity detector operating on $x_{p}(k)$. It might seem reasonable that estimating ERLE should be omitted in a doubletalk situation, (i.e., when near-end and far-end speech are present simultaneously). However, this is not necessary. The value of $g(k)$ during doubletalk is not significant, since during doubletalk, convergence of the background filter is not possible anyway. The only concern is that the present averaging functions are defined so that the ERLE estimate is allowed to converge to its proper value reasonably fast after the end of a doubletalk session.

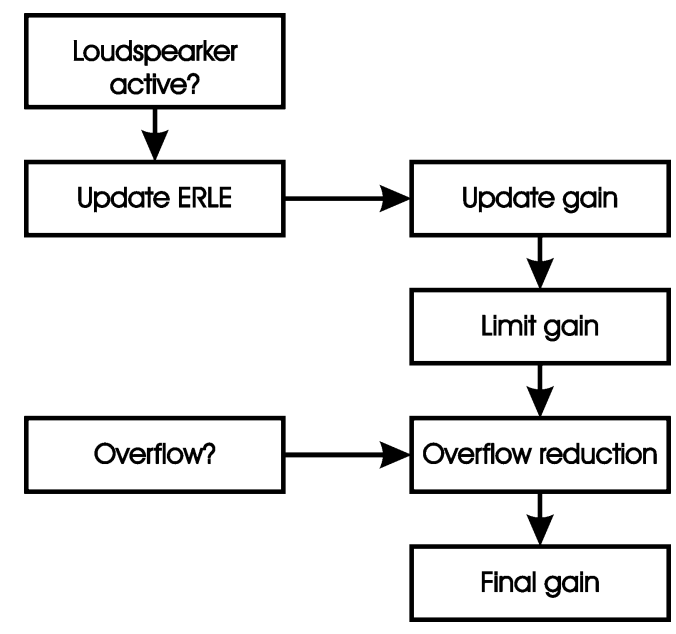

Fig. 3. Flowchart illustrating the control of the adaptive gain $g(k)$.

Increasing the value of $g(k)$ above a certain limit is useless since when the least-significant bits of the foreground filter coefficients have converged, no further improvement of the output signal $e_{f_{p}}(k)$ (see Fig. 2) can be achieved.

When the value of $g(k)$ changes, the coefficients of the background filter needs to be shifted correspondingly (i.e., if $g(k)$ is increased, the filter coefficients need to be upshifted with the corresponding value). To avoid an increase in peak complexity, the background filter update can be omitted when the background filter coefficients need to be shifted. The value of $g(k)$ should only be allowed to change at certain intervals to avoid a reduction in convergence.

At all times, the background filter update factor $\mu e_{b_{p}}(k) /\left(\left\|\mathbf{x}_{p}(k)\right\|^{2}+\epsilon\right)$ must be prevented from overflowing. If the filter update factor overflows, $g(k)$ should be reduced to a sufficiently low value.

The proposed control algorithm, depicted in Fig. 3, is thus as follows: The ERLE is estimated through

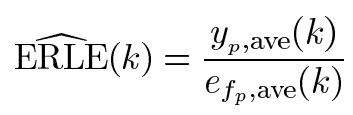

where the averages $y_{p}$,ave $(k)$ and $e_{f_{p}}$,ave $(k)$ are defined through

$$
y_{p}, \text { ave }(k)=(1-\gamma) y_{p}, \text { ave }(k-1)+\gamma y_{p}^{2}(k)
$$

where $\gamma$ is an averaging constant.

Far-end speech activity can be detected by $\left\|\mathbf{x}_{p}(k)\right\|^{2}$ with a threshold $T_{x}$ and declaring $x_{p}(k)$ active whenever

$$
\left\|\mathbf{x}_{p}(k)\right\|^{2}>T_{x}
$$

The gain $g(k)$ should be increased or decreased depending on the value of $\operatorname{ERLE}(k)$. In order to reduce complexity, this is performed at every $M$ sample (i.e., the same interval as the update check)

$$
g(k)= \begin{cases}g(k-1)+1, & \text { if } g(k) \leq K \log _{2}(E \widehat{R L} E(k)) \\ g(k-1)-1, & \text { otherwise }\end{cases}
$$


where $K$ is a fixed parameter determining how much the foreground filter must converge before the gain $g(k)$ can be increased. Increasing the gain $g(k)$ over a certain limit will not improve the performance. In fact, a very large $g(k)$ might result in an absent bit-range overlap between the foreground and background filter. Thus, $g(k)$ should be limited according to

$$
g(k)=L \quad \text { if } \quad g(k)>L
$$

where $L$ is the limiting factor.

At every sample, the value of the update factor is checked for possible overflow, i.e., checking if

$$
\frac{\left|\mu e_{b_{p}}(k)\right|}{\left\|\mathbf{x}_{p}(k)\right\|^{2}+\epsilon}>1 \text {. }
$$

If the update factor has overflowed, the value of $g(k)$ is reduced as

$$
g(k)=g(k-1)-\mathcal{R}\left(\log _{2}\left(\frac{\left|\mu e_{b_{p}}(k)\right|}{\left\|\mathbf{x}_{p}(k)\right\|^{2}+\epsilon}\right)\right)
$$

where $\mathcal{R}(\cdot)$ denotes a roundoff operation which rounds to the nearest integer toward infinity.

If $g(k)$ is changed, either from (27) or (30), the error $e_{b_{p}}(k)$ is set to zero and the update of the background filter is omitted. Instead, the background filter is modified as

$$
\mathbf{w}_{b_{p}}(k)=2^{g(k)-g(k-1)} \mathbf{w}_{b_{p}}(k) .
$$

The described two-path solution as depicted in Fig. 2 and defined in (13)-(15), (17)-(22), and (24)-(31) is denoted as the proposed two-path algorithm.

\section{COMPLEXITY}

Equations (13), (15), and (20)-(22) are performed in the conventional two-path solution as well, so they imply no extra complexity. When (17), (18), (27), (28), (30) and (31) are performed, the background filter update is omitted so executing these equations does not add any peak complexity. Equation (14) requires 1 extra multiplication, (24) requires 1 division, (25) requires 3 multiplications and 1 addition, (26) requires 1 comparison, and, finally, (29) requires 1 comparison. The total number of extra complexity required by the proposed algorithm is thus 4 multiplications, 1 division, 1 addition, and 2 comparisons.

The filtering processing equations (13) and (15) together require $2 N$ multiplications and additions. The NLMS update (21) requires a little more than $N$ multiplications and additions. In acoustic echo cancellation, typically $N>1000$. Thus, comparing the extra complexity introduced by the proposed algorithm with the filtering and NLMS update operations shows that the increase in complexity is insignificant.

\section{Simulations}

In order to illustrate and verify the performance of the proposed two-path algorithm, several simulations were performed. In the simulations, bandlimited flat spectrum noise and speech signals were used as an input signal $x(k)$. The microphone signal $y(k)$ was obtained through

$$
y(k)= \begin{cases}\mathbf{x}(k)^{T} \mathbf{h}_{1}+n(k), & \text { if } k<I \\ \mathbf{x}(k)^{T} \mathbf{h}_{2}+n(k), & \text { otherwise }\end{cases}
$$

TABLE I

DEFAULT PARAMETER SETTINGS

\begin{tabular}{|r|l|}
\hline Parameter & Value \\
\hline$N$ & 1200 \\
$\mu$ & 0.5 \\
$\epsilon$ & 4 \\
$q$ & 12 \\
$\gamma$ & 0.01 \\
$T_{x}$ & 5 \\
$K$ & 1 \\
$L$ & 8 \\
ENR & 35 \\
\hline
\end{tabular}
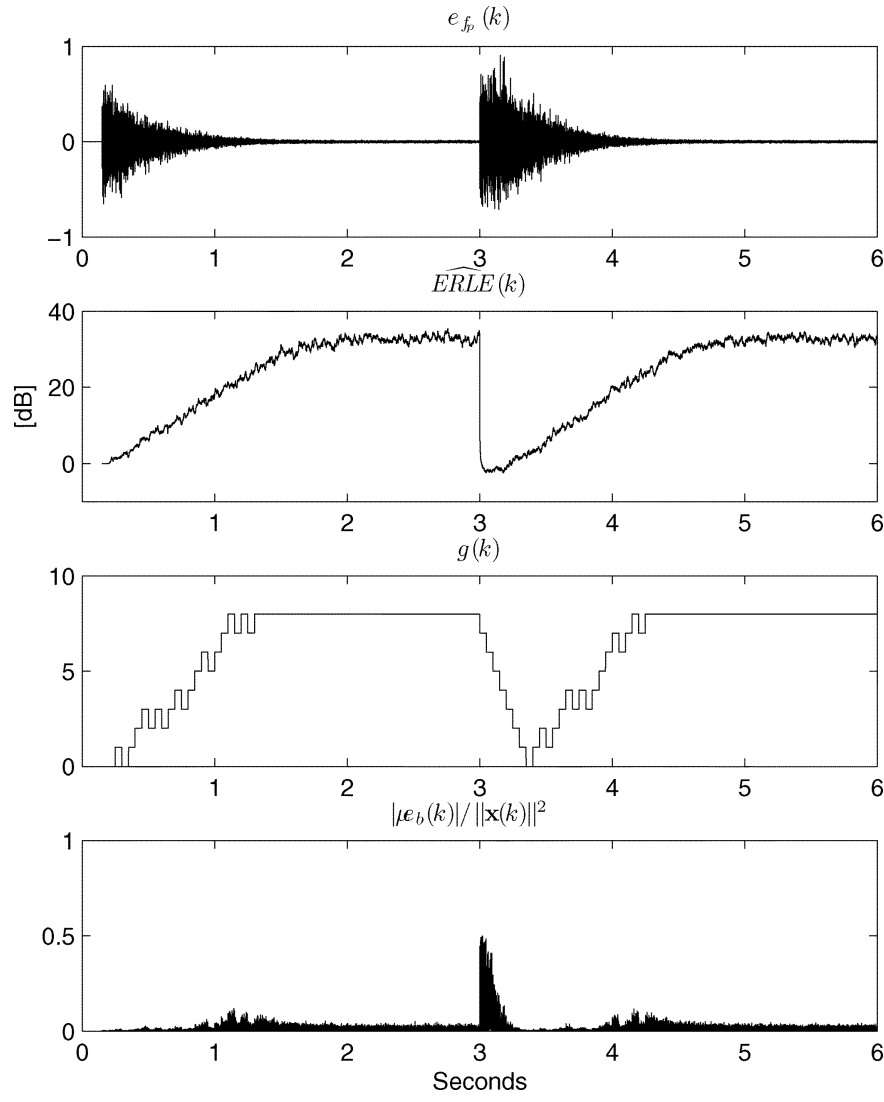

Fig. 4. Error signal $e_{f_{p}}(k)$, the ERLE estimation $E \widehat{R L} E(k)$, the gain factor $g(k)$, and the update factor $\left|\mu e_{b_{p}}(k)\right| /\left\|\mathbf{x}_{p}(k)\right\|^{2}$ of the proposed algorithm for an echo path change situation.

where $\quad \mathbf{h}_{1}=\left[h_{1,0}, \ldots, h_{1, N-1}\right]^{T}$ and $\mathbf{h}_{2}=$ $\left[h_{2,0}, \ldots, h_{2, N-1}\right]^{T}$ are FIR models of two different LEM systems corresponding to two different spatial positions of the microphone, $I$ is a parameter controlling at which time instant the echo path change occurs, and $n(k)$ is the background noise. The purpose of this echo path change is to verify that the gain control of the proposed solution properly handles situations where the echo cancellation performance suddenly changes. The background noise $n(k)$ was bandlimited flat spectrum noise with its level defined by the echo-to-noise ratio (ENR). The sampling frequency was set to $8 \mathrm{kHz}$. Default parameters are shown in Table I. In some simulations (see Figs. 7-9), some of these settings were altered.

In Fig. 4, the behavior of the proposed algorithm is shown for an echo-path change situation, where a bandlimited flat spectrum noise signal is used as an input signal. Fig. 4 demonstrates 


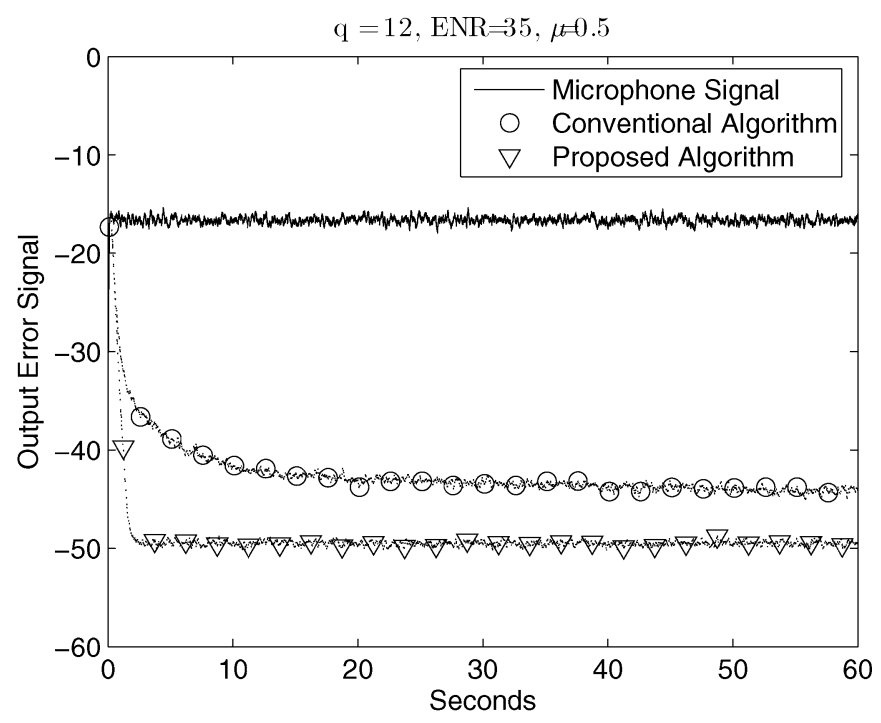

$\mathrm{q}=12, \mathrm{ENR} 35, \mu-0.5$

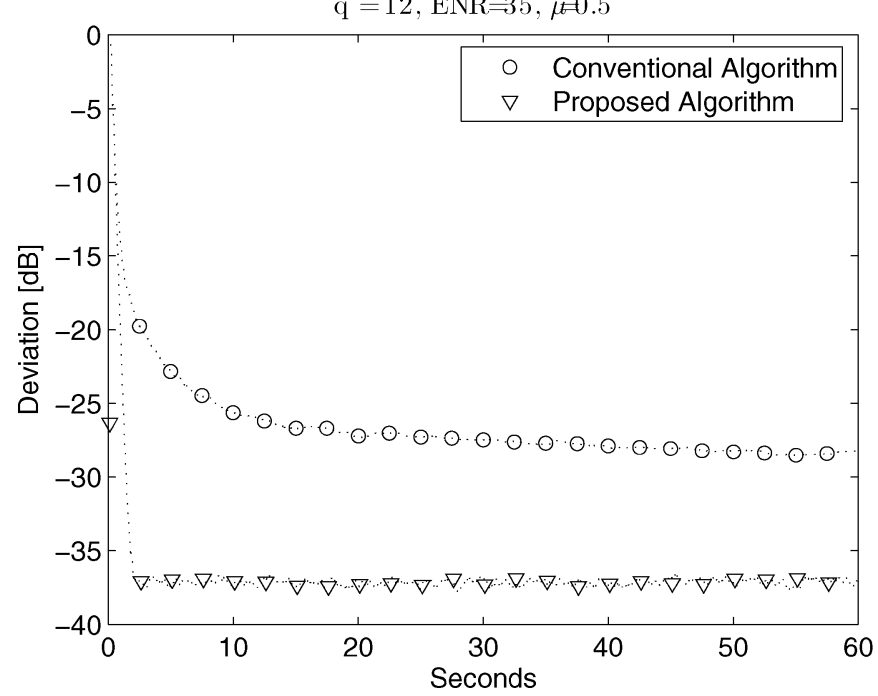

Fig. 5. Upper plot shows the output error signals of the conventional and the proposed two-path algorithm in a comparison using bandlimited flat spectrum noise as the input signal. The microphone signal is also shown for comparison. Lower plot: Filter deviations of the conventional and the proposed two-path algorithm for the same comparison as in the upper plot.

how the $g(k)$ signal follows the estimated ERLE and that the update factor is kept below 1.

The performance of the two solutions was evaluated through average squared error (i.e., the average of $e_{f}^{2}(k)$ and $e_{f_{p}}^{2}(k)$ ), as well as through filter deviation (system distance) [9], [10]. The deviation is measured as the normalized squared deviation, that is, the deviation $D_{\mathbf{w}_{f}}(k)$ of filter $\mathbf{w}_{f}(k)$ from the impulse response $\mathbf{h}_{j}$ is given by

$$
D_{\mathbf{w}_{f}}(k)=\frac{\sum_{i=0}^{N-1}\left(h_{j, i}-w_{f, i}(k)\right)^{2}}{\left\|\mathbf{h}_{j}\right\|^{2}} .
$$

The deviation $D_{\mathbf{w}_{f_{p}}}(k)$ of filter $\mathbf{w}_{f_{p}}(k)$ is calculated in the same manner. Fig. 5 depicts the performance of the proposed and the conventional two-path algorithm when bandlimited flat spectrum noise is used as an input signal and Fig. 6 when a speech signal is used. Parameter settings as given in Table I were used
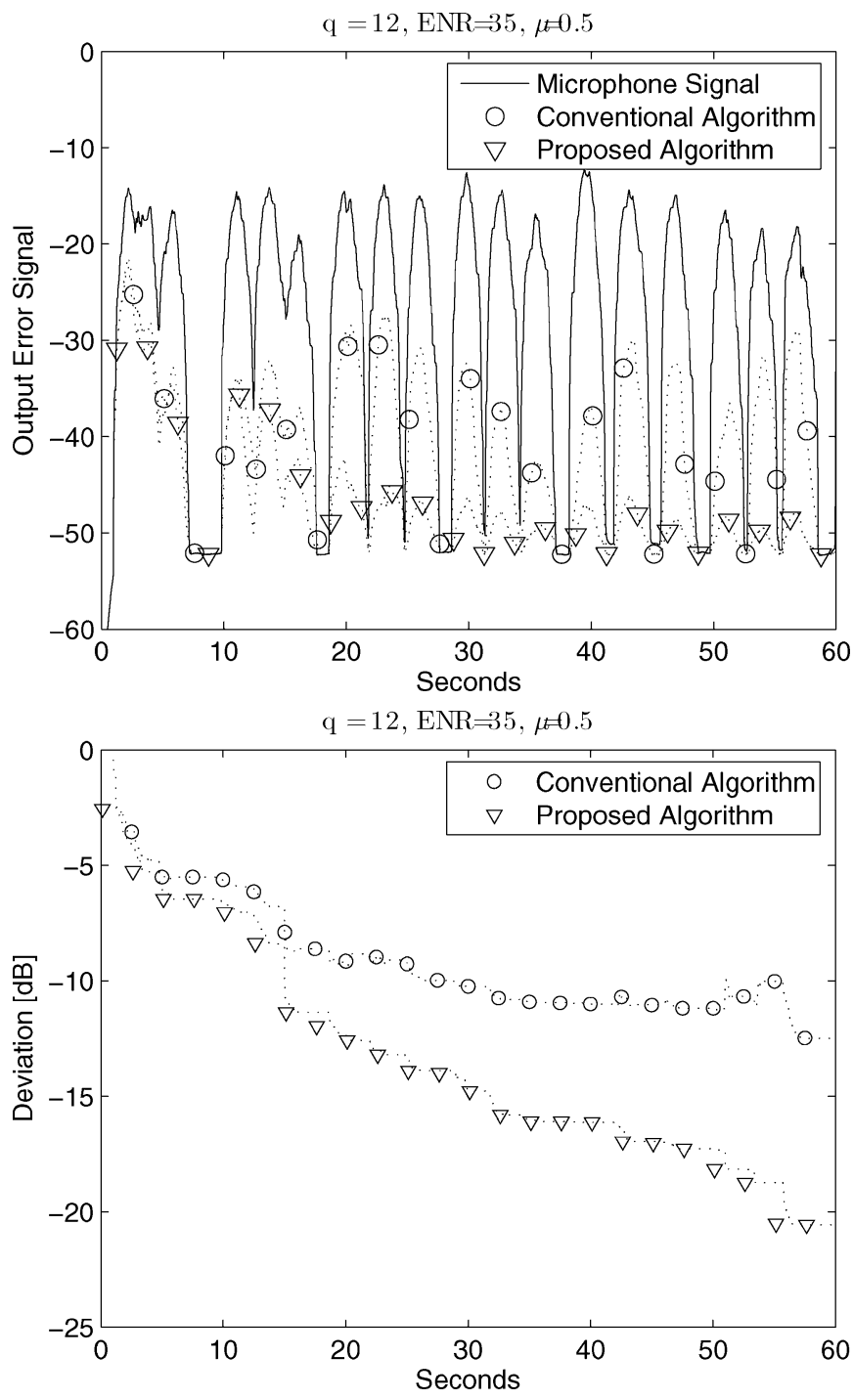

Fig. 6. Upper plot shows the output error signals of the conventional and the proposed two-path algorithm in a comparison using speech as an input signal. The microphone signal is also shown for comparison. The lower plot shows the filter deviations of the conventional and the proposed two-path algorithm for the same comparison as in the upper plot.

in the generation of both figures. The figures demonstrate how the convergence performance can be improved by employing the proposed algorithm.

Figs. 7-9 show the filter deviations, using the bandlimited flat spectrum noise as an input signal, for a number of different settings of the bit precision $q$, the echo-to-noise ratio ENR, and the step-size control parameter $\mu$.

Fig. 7 shows that for bit precisions of $q=8$ and $q=12$, the performance of the proposed algorithm is significantly better than that of the conventional algorithm. For $q=16$, there is still an improvement, but it is more moderate. This demonstrates that the performance of the proposed and the conventional two path will be similar if the bit precision is increased to a level where the NLMS in the conventional two-path algorithm no longer suffers from finite precision effects.

Fig. 8 demonstrates how the improvement is dependent on the background noise level. It is apparent that for no background noise (i.e., ENR = inf or a "normal" ENR of $35 \mathrm{~dB}$ ), the 

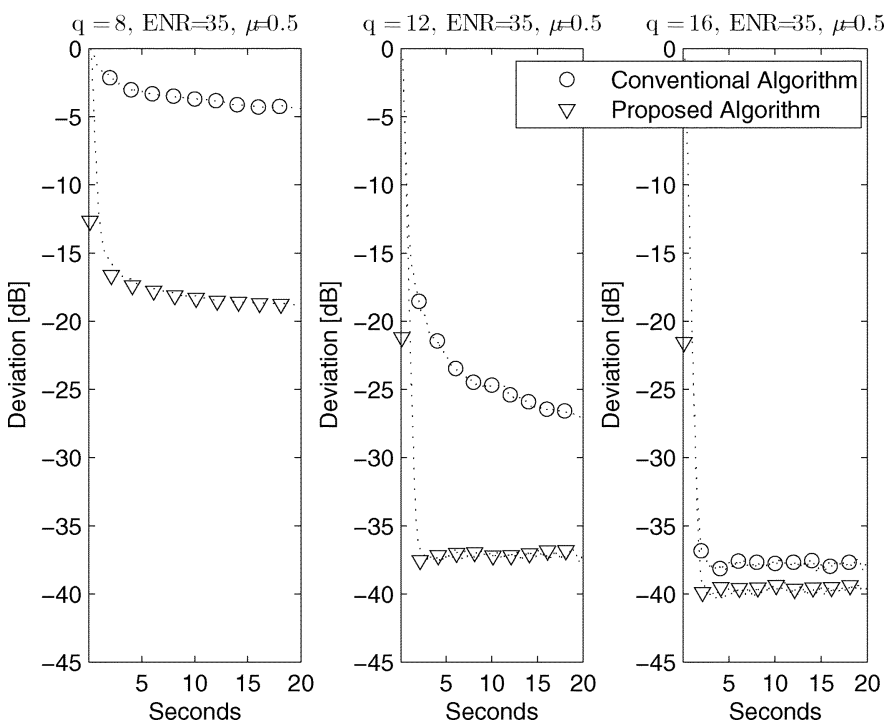

Fig. 7. Filter deviation of the proposed and the conventional two-path NLMS for different values of the bit precision parameter $q$.
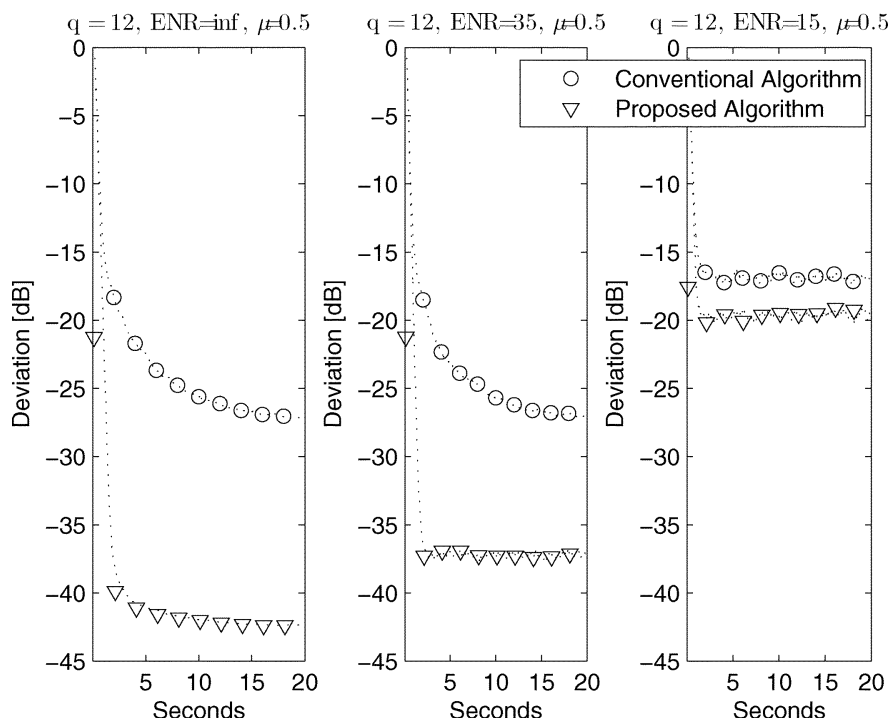

Fig. 8. Filter deviation of the proposed and the conventional two-path NLMS for different values of the echo-to-noise ratio (ENR).

proposed two-path algorithm gives a significant improvement over the conventional scheme. For a "high" noise level (i.e., $\mathrm{ENR}=15 \mathrm{~dB}$ ), the impairments of the background noise are to make quantization effects less significant and the two algorithms have similar performances.

Fig. 9 shows deviation curves for different settings of the step-size control parameter $\mu$. For the conventional two-path, the figure shows that when $\mu$ is reduced from 1 to 0.5 , the deviation after $20 \mathrm{~s}$ is reduced from $-24 \mathrm{~dB}$ to $-27 \mathrm{~dB}$. Further reduction of $\mu$ to $\mu=0.25$, however, yields an increase of deviation to $-22 \mathrm{~dB}$, see Fig. 9. This demonstrates the well-known fact that by reducing the step-size in finite precision arithmetic might not lead to increased steady-state echo cancellation performance (e.g., [15]).

The proposed algorithm does not suffer from quantization effects, instead it is the background noise that hinders further
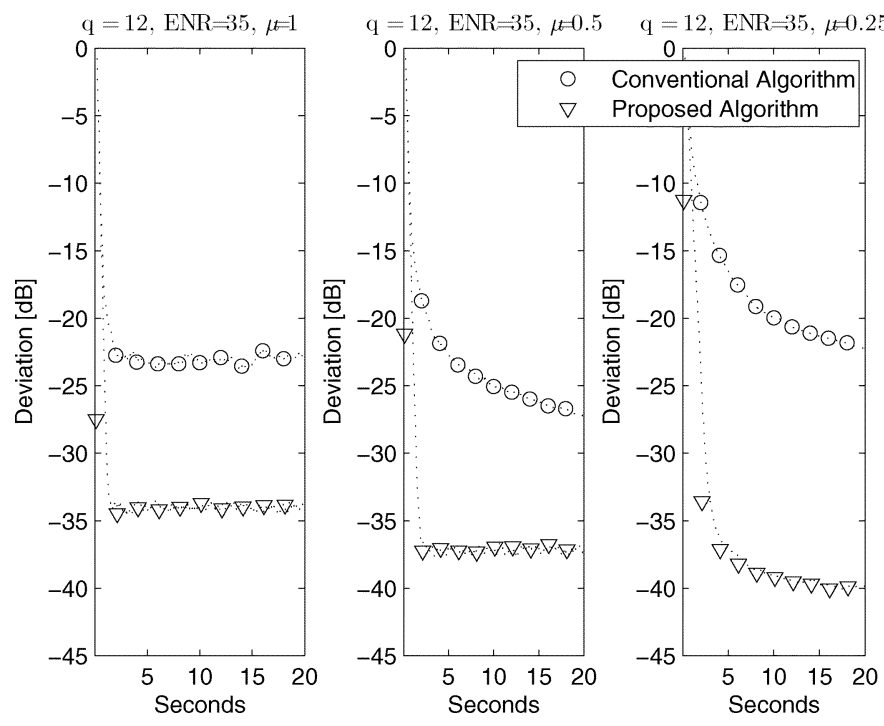

Fig. 9. Filter deviation of the proposed and the conventional two-path NLMS for different values of the step-size control parameter $\mu$.

convergence. Therefore, for the proposed algorithm, the steadystate performance is improved as $\mu$ is reduced, as can be seen in Fig. 9.

\section{CONCLUSION}

The fixed-point adaptive filters suffer more or less from stalling. Previous solutions to this problem have consisted of setting the lowest limit of the adaptive filter step size or to increase the number of bits. This paper has proposed a modification of the traditional two-path adaptive filter solution for finite precision implementations, which reduces the impact of finite precision effects, such as stalling. The modification uses redundance capacity in the filter computations to reduce the effects of quantization and, thus, an insignificant increase in computational complexity is implied. If the effects of quantization are significant in relation to other noise contributions, the increased echo cancellation performance of the proposed method is evident. This was demonstrated in simulations using both flat spectrum bandlimited noise as well as speech signals.

\section{REFERENCES}

[1] K. Ochiai, T. Araseki, and T. Ogihara, "Echo canceler with two echo path models," IEEE Trans. Commun., vol. COM-25, no. 6, pp. 8-11, Jun. 1977.

[2] J. Liu, "A novel adaption scheme in the NLMS algorithm for echo cancellation," IEEE Signal Process. Lett., vol. 8, no. 1, pp. 20-22, Jan. 2001.

[3] F. Lindstrom, M. Dahl, and I. Claesson, "The two-path algorithm for line echo cancellation," in Proc. IEEE Tencon, Nov. 2004, pp. 637-640.

[4] Y. Haneda, S. Makino, J. Kojima, and S. Shimauchi, "Implementation and evaluation of an acoustic echo canceller using the duo-filter control system," in Proc. IWAENC, Jun. 1995, pp. 79-82.

[5] S. Shimauchi, S. Makino, Y. Haneda, A. Nakagawa, and S. Sakauchi, "A stereo echo canceller implemented using a stereo shaker and a duo-filter control system," in Proc. IEEE ICASSP, 1999, vol. 2, pp. 857-860.

[6] T. Gansler, J. Benesty, and S. L. Gay, Acoustics Signal Processing for Telecommunication. Norwell, MA: Kluwer, 2000.

[7] R. L. Bouquin-Jeannes and G. Faucon, "Control of an adaptive echo canceller using a near-end speech detector," Signal Process., vol. 81, no. 3, pp. 483-489, 2001.

[8] A. Mader, H. Puder, and G. U. Schmidt, "Step-size control for acoustic cancellation filters-An overview," Signal Process., vol. 80, no. 9, pp. $1697-1719,2000$. 
[9] E. Hansler and G. Schmidt, Acoustic Echo and Noise Control a Practical Approach. Hoboken, NJ: Wiley, 2004.

[10] S. Haykin, Adaptive Filter Theory, 4th ed. Upper Saddle River, NJ: Prentice-Hall, 2002.

[11] R. D. Gitlin, J. E. Mazo, and M. G. Taylor, "On the design of gradient algorithms for digitally implemented filters," IEEE Trans. Circuit Theory, vol. CT-20, no. 2, pp. 125-136, Mar. 1973.

[12] C. Caraiscos and B. Liu, "A roundoff error analysis of the LMS adaptive algorithm," IEEE Trans. Acoust., Speech Signal Process., vol. ASSP-32, no. 1, pp. 34-41, Feb. 1984.

[13] N. J. Bershad and J. C. M. Bermudez, "New insights on the transient and steady-state behavior of the quantized LMS algorithm," IEEE Trans. Signal Process., vol. 44, no. 10, pp. 2623-2625, Oct. 1996.

[14] N. J. Bershad and J. C. M. Bermudez, "A non-linear analytical model for the quantized lms algorithm-The power-of-two step size case," IEEE Trans. Signal Process., vol. 44, no. 5, pp. 2895-2901, May 1996.

[15] J. C. M. Bermudez and N. J. Bershad, "Transient and tracking performance analysis of the quantized LMS algorithm for time-varying system identification," IEEE Trans. Signal Process., vol. 44, no. 8, pp. 1990-1996, Aug. 1996.

[16] F. Lindstrom, M. Dahl, and I. Claesson, "A finite precision LMS algorithm for increased quantization robustness," in Proc. IEEE ISCAS, May 2003, pp. 365-368.

[17] D. E. Knuth, The Art of Computer Programming: Seminumerical Algorithms, 2nd ed. Reading, MA: Addison-Wesley, 1989.

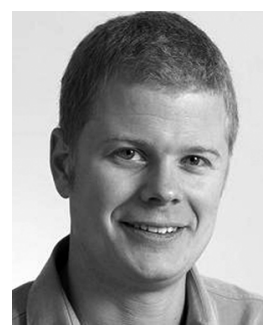

Fredric Lindström (M'05) was born in Skelleftea, Sweden. He received the M.Sc. degree in applied physics from Uppsala University, Uppsala, Sweden, in 2001.

Since 2000, he has been with Department of Research and Development, Konftel AB, Umea, Sweden, where he was appointed Software Development Manager in 2004. His current research interest is adaptive signal processing with applications in hands-free systems (e.g., acoustic echo canceling, rithms for finite precision implementations)

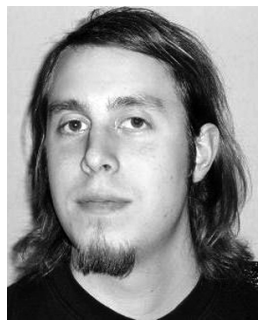

Christian Schüldt (S'07) was born in Stockholm, Sweden. He received the B.Sc. degree in electrical engineering with a specialization in mobile communication from the Royal Institute of Technology, Stockholm, Sweden, in 2001 and the M.Sc. degree in electrical engineering with a specialization in signal processing and computer vision from the Royal Institute of Technology, Stockholm, in 2004.

Currently he is a Research Engineer with the Department of Signal Processing, Blekinge Institute of Technology, Ronneby, Sweden. His areas of professional interest include signal processing techniques and real-time programming.

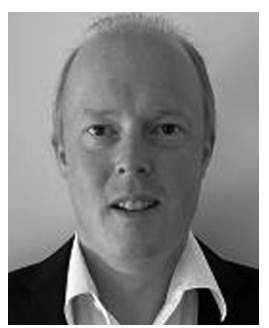

Mikael Långström received the M.Sc. degree from Umeå University, Umea, Sweden, in 1999. He was a Development Engineer with Fält Communication, Umea, in 2000 working on the development of new products. He holds several patents.

Mr. Långstrom was appointed CTO in 2003 and in 2007, was appointed CEO of Fält Communication.

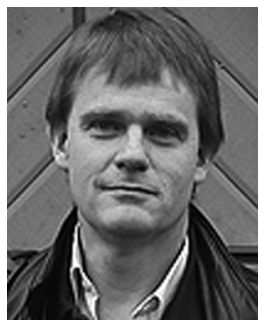

Ingvar Claesson (M'91) received the Ph.D. degree from Lund University, Lund, Sweden, in 1986.

In 1992, he was appointed Associate Professor at Lund University. Since 1998, he has been the Chair of Signal Processing at the Blekinge Institute of Technology, Ronneby, Sweden. In 1990, he was one of the founders of the Department of Signal Processing, Blekinge Institute of Technology, where he is currently Head of Research and Principal Supervisor of Signal Processing. His current research interests are adaptive signal processing, blind equalization, adaptive beamforming, speech enhancement, active noise control, filter design, and antenna arrays. 